\title{
ARTEFATOS SEMÂNTICOS DE NEGÓCIO APLICADOS AO CONTEXTO DE EMPRESA INTEGRADA
}

William Brepohl (william brepohl@hotmail.com) - Universidade Tecnológica Federal do Paraná (UTFPR).

Gabriel Magron Klockner (gabriel.klockner@gmail.com) - Universidade Tecnológica Federal do Paraná (UTFPR).

Caio Alexandre Soares Fabrão (caiofabrao@alunos.utfpr.edu.br) - Universidade Tecnológica Federal do Paraná (UTFPR).

Kaio Vasconcelos de Oliveira (kaiooliveira@alunos.utfpr.edu.br) - Universidade Tecnológica Federal do Paraná (UTFPR).

Milton Borsato (borsato@utfpr.edu.br) - Universidade Tecnológica Federal do Paraná (UTFPR).

\section{RESUMO}

A integração entre vários parceiros de negócios é uma característica essencial para o desenvolvimento totalmente nos dias atuais. Uma das maneiras de permitir que isso ocorra é por meio da modelagem de processos de negócios. No entanto a troca de informações comerciais entre duas empresas diferentes pode se mostrar mais complicado do que parece, pois problemas com falta de padronização, inconsistência de dados e interpretações ambíguas são frequentes. Uma maneira de se integrar e obter sucesso é o uso de modelos semânticos, mas há falta de estudos que demonstrem o real benefício de utilizar na prática. O objetivo deste trabalho é verificar o uso das técnicas e as vantagens para se utilizar os Artefatos de Negócios com maior capacidade semântica. Com à sua expressividade semântica, esses modelos permitem grandes melhorias no contexto dos padrões de interoperabilidade, especialmente em cenários onde as relações de classificação são importantes e várias restrições precisam ser aplicadas às informações do modelo.

Palavras chave: Integração Empresarial, B2B, Artefatos de Negócios, OWL, Prova de Conceito 


\section{INTRODUÇÃ̃}

A competição por mercado tem feito com que empresas busquem maneiras para aprimorar seus processos internos. A utilização de modelagem de processos de negócios tem sido um método fundamental para as empresas. É definido como uma descrição estruturada de diversos aspectos considerados relevantes para a empresa (ROSPOCHER et al., 2008). Por meio destes modelos os recursos organizacionais podem ser estudados e otimizados para alcançar os objetivos estratégicos ou operacionais da empresa (GASSEN, 2014). Através desta modelagem é possível reduzir consideravelmente inconsistências e redundâncias nos processos empresariais.

O modelo empresarial é definido como um conjunto de modelos de processos internos de uma empresa. Anteriormente apenas dois aspectos eram considerados: os processos e atividades e o domínio de negócio que a empresa opera. Recentemente outras propriedades da empresa como objetivos, recursos humanos, regras, entre outras, também se tornaram importantes para a construção do modelo empresarial (ROSPOCHER et al., 2008).

Uma das iniciativas que objetivam facilitar a integração entre as modelagens dos processos empresariais em diferentes organizações é o conceito de Artefatos de Negócio. Os modelos tradicionais têm um enfoque nos passos realizados em cada processo do negócio e nas interações que vão transformando dados e informações através destes processos. A modelagem através de Artefatos de Negócio busca identificar inicialmente os artefatos que são relevantes para cada processo do negócio e só então especificar como estes "objetos" são alterados ao longo do negócio (LIU; BHATTACHARYA; WU, 2007).

Mesmo fazendo uso de artefatos de negócio, a modelagem pode ser passível de ambiguidades quando a linguagem utilizada dá margem para tal. Modelos onde a capacidade de expressividade é fraca podem ser mais simples e úteis para a compreensão humana. Porém não são suficientes para suportar a interoperabilidade automatizada e nem para fazer inferências aprofundadas sobre seu conteúdo (ALMEIDA, 2006).

Uma forma de representação destes modelos é através do uso de ontologias formais. Ontologias são estruturas bastante adequadas para a representação de artefatos de negócio, pois, através de sua expressividade semântica, elas são capazes de definir atributos e relações com grau de formalidade variável. $\mathrm{O}$ uso de ontologias formais apresenta um conjunto de axiomas completos que restringem as ambiguidades de interpretação permitindo inferências mais complexas sobre o modelo (ALMEIDA, 2006). 
Uma vez evidenciada a importância da modelagem dos artefatos empresariais, o uso de ontologias para tal e identificado seu potencial para facilitar e permitir a integração inteligente de processos e informações num contexto de interoperabilidade de negócios, é que este trabalho buscou o seguinte objetivo: Investigar a viabilidade técnica da utilização de artefatos semânticos de negócio como alternativa à solução não-semântica para o problema de interoperabilidade no contexto de integração de empresas.

\section{REVISÃO TEÓRICA}

\subsection{Interoperabilidade entre Sistemas}

Interoperabilidade entre sistemas pode ser definida pela capacidade dos sistemas operacionais operarem e cooperarem. Mesmo na presença de diferentes representações de dados e protocolos de comunicação de modo que esta interação obtenha os resultados conforme esperado (CAFEZEIRO et al., 2008; KAMADA, 2011).

Para que dois ou mais sistemas computacionais sejam interoperáveis, é importante que a troca de dados entre eles tenha o mínimo de ambiguidade possível. Assim garantindo que os dados recebidos por um sistema sejam interpretados exatamente da mesma forma como eram interpretados pelo sistema emissor (KAMADA, 2011). Existem dois grandes desafios para a interoperabilidade: o primeiro é relacionado aos conflitos de semântica, e o segundo é o trabalho envolvido para se desenvolver tradutores entre modelos distintos (CIOCOIU; NAU; GRUNINGER, 2001).

$\mathrm{Na}$ interoperabilidade três aspectos devem ser considerados: semântico, sintático e estrutural (LIMA; CARVALHO, 2004). A semântica permite compreender o significado de cada elemento, e as associações da qual o elemento faz parte. É necessário o uso de vocabulários específicos como ontologias e padrões de metadados (KAMADA, 2011). A sintática como os metadados devem ser codificados para a transferência de informações. Na web, por exemplo, a XML é uma das principais tecnologias responsáveis por garantir este tipo de interoperabilidade. A estrutural determina como os recursos estão organizados, além de tratar dos tipos envolvidos e os possíveis valores de cada tipo. 


\subsection{Integração de Processos de Negócio}

Processos de negócio é definido como um conjunto estruturado de atividades relacionadas através de uma sequência lógica, com começo e fim bem definidos, executados para alcançar um objetivo específico (NETO; JUNIOR, 2008).

Em uma organização existem alguns recursos que são passíveis de modelagem como os dados, as informações e o conhecimento existente. Um modelo organizacional como qualquer outro modelo pode ser representado por uma linguagem. O conhecimento inserido em um modelo organizacional representa aspectos relevantes e permite que os processos possam ser compreendidos (ALMEIDA, 2006).

O mesmo processo de negócio pode ser implementado de diversas formas em diferentes organizações. Muitas organizações crescem através de fusões e aquisições de novas empresas, cada unidade possui suas próprias particularidades e formas de representar as informações relacionadas aos processos. Quando um canal de comunicação é criado entre essas unidades, é comum que apareçam redundâncias e inconsistências, principalmente quando se trata de grandes empresas. Por isso é importante que a integração seja implementada com planejamento, pois ela pode trazer ganhos importantes, como uma maior facilidade de estabelecer novos canais de comunicação com outros sistemas, além de uma redução significativa nos custos operacionais da empresa (LIU; BHATTACHARYA; WU, 2007).

Existe ainda neste tipo de integração, a complexidade gerada por se utilizar diferentes linguagens na modelagem de processo e no fato de que a transformação da informação de um modelo para o outro pode gerar ambiguidades semânticas nas representações do negócio. Mas, de acordo com Liu, Bhattacharya e Wu (2007), o verdadeiro problema está em comunicar a intenção por meio de modelos de processos de negócios, questão que é fundamental e independente da semântica adotada na modelagem.

Um modelo de processo representa a documentação de um processo de negócio, descrita através de uma notação ou linguagem (GASSEN, 2014). A modelagem de processos de negócio tem por objetivo definir uma abstração de como o negócio funciona (OLIVEIRA, 2010).

A modelagem de processos de negócios é uma ferramenta indispensável para empresas que desejam modelar a lógica e os objetivos de seus negócios. Um modelo de processo de negócio serve para representar as ações realizadas por atores referentes ao negócio utilizando 
seus recursos para alcançar determinados objetivos da organização. Além disso, modelos de processos de negócios também podem ser usados para realizar a comunicação entre diferentes atores envolvidos em um negócio (LIU; BHATTACHARYA; WU, 2007).

\subsection{Artefatos de Negócio}

Os modelos tradicionais de processo concentram-se nas ações tomadas para alcançar determinado objetivo. Outra possível abordagem é focar no que é posto em prática, como descrever as operações identificando os objetos relevantes para o negócio (por exemplo, Pedido de Compra) e como estes são processados para atingir determinado objetivo. Estes objetos relevantes são chamados de Artefatos de Negócio (LIU; BHATTACHARYA; WU, 2007).

Os processos de negócio podem ser divididos em duas categorias diferentes: espaço contextual e espaço comportamental (LIU; BHATTACHARYA; WU, 2007). O espaço contextual manifesta-se nos próprios artefatos de negócios. Já o espaço comportamental manifesta-se em todas as atividades que o negócio executa. Na modelagem de processo tradicional a ênfase é sobre o espaço comportamental; os aspectos contextuais são definidos como atributos de dados de entrada e saída das atividades de trabalho. Na modelagem operacional os aspectos contextuais e comportamentais são tratados com a mesma ênfase, cada tarefa de trabalho é definida levando em consideração o artefato de negócio em que a tarefa opera (LIU; BHATTACHARYA; WU, 2007).

Um artefato de negócio é um artefato identificável e auto descritivo. Ele possui um atributo que o identifica unicamente dentro de uma organização. Por carregar consigo essa propriedade de unicidade pode-se dizer que um artefato não pode ser dividido. Um artefato de negócio é auto descritivo no sentido que seus atributos são nomeados conforme sua utilização em determinado domínio do negócio. As informações contidas nos artefatos podem ser representadas usando pares nome-valor. O modelo de informação de um artefato pode ser modelado utilizando qualquer linguagem de modelagem adequada, como um diagrama entidade-relacionamento ou uma XML (LIU; BHATTACHARYA; WU, 2007).

\subsection{Ontologia}

Na filosofia, ontologia é a ciência que estuda o "ser" e suas propriedades (FONSECA; EGENHOFER; BORGES, 2000). Pesquisadores da área de Inteligência Artificial (IA) e Web adaptaram o termo de acordo com sua realidade (SOUZA; ALVARENGA, 2004). Para esses 
pesquisadores, ontologias são teorias que especificam um vocabulário relativo a um certo domínio. Este vocabulário define entidades, classes, propriedades, predicados e funções e as relações entre esses (FONSECA; EGENHOFER; BORGES, 2000).

No contexto de IA e Web, ontologias são modelos de representação de entidades e suas relações, centradas em um domínio específico do conhecimento ou de alguma atividade. A ideia por trás de utilizar ontologias está na necessidade de se ter um vocabulário capaz de permitir a troca de informações entre os membros de uma comunidade, sejam eles humanos ou agentes inteligentes (SOUZA; ALVARENGA, 2004).

De acordo com Gassen (2014), às palavras utilizadas por nomear os elementos de um modelo de processos de negócio são suscetíveis à ambiguidade, fator que pode resultar em perda de qualidade. Alguns estudos citados em Gassen (2014) propõem a utilização de ontologias para aprimorar modelos de processos de negócio, inclusive aumentando sua capacidade de interoperabilidade. Esse tipo de estrutura juntamente com a semântica de modelos é adequado para representar situações do mundo real (CAFEZEIRO et al., 2008) e suportar o contexto de interoperabilidade.

Existem diversas linguagens que implementam soluções ontológicas, sendo a Web Ontology Language (OWL) uma das mais utilizadas. A OWL foi criada e é mantida pela World Wide Web Consortium (W3C), uma comunidade internacional para o desenvolvimento de padrões abertos para a internet (W3C, 2015). É uma linguagem computacional baseada em lógica, que pode ser compreendida por programas computacionais (W3C, 2001). A OWL busca representar uma parte do universo em que está trabalhando, sendo ele representado por classes, propriedades, instâncias e axiomas. O principal foco da OWL é a extração de conhecimento, possibilidade de raciocínio e inferência, bem como capacidade de identificação e marcação de elementos (ROSPOCHER et al., 2008).

\section{METODOLOGIA}

Como o objetivo deste trabalho era realizar a comparação entre a utilização de duas técnicas para modelagem de negócios, utilizando modelos semântico (MSAN) e modelos não semânticos (MNSAN). Para isso realizou uma entrevista com Marketplaces e com lojistas, onde foi disponibilizado dois arquivos um em formato PHP e outro em XML. Com base nas informações coletadas foi criado um padrão genérico com o intuito de atender as necessidades 
do contexto de realizar a troca de informações entre os parceiros de negócios sobre produtos de moda. Neste padrão genérico é definido as propriedades e as informações necessárias.

O MSAN corresponde a um modelo de Artefato de Negócio que possui alguma capacidade semântica, além da representação meramente sintática. O MSAN busca focar na transformação dentro dos processos de negócio, ao invés de somente nos processos. A capacidade semântica de um MSAN permite que uma gama maior de propriedades sejam descritas no próprio modelo, como relações entre artefatos, restrições sobre seus atributos, relações de classificação e outras tantas não citadas (LIU; BHATTACHARYA; WU, 2007).

Para a construção do MSAN foi escolhida a OWL 2, utilizando a ferramenta de software Protégé, na sua versão 4.3.0, para criar e modificar as ontologias. Foram identificados os conceitos e propriedades presentes no modelo conceitual genérico e categorizá-los em classes, propriedades de dados, propriedades de objetos ou indivíduos.

O MNSAN é a forma oposta ao MSAN, corresponde à forma tradicional de modelo de um Artefato de Negócio. Possui propriedades de representação única por meio de identificadores e atributos que podem ser representados usando pares nome-valor (LIU; BHATTACHARYA; WU, 2007). Não possui considerável expressividade semântica, mas restringe-se a um artefato meramente sintático e restrições aplicadas quase somente à sua sintaxe, ou seja, passível de maior ambiguidade (HERAVI, 2012; GASSEN, 2014).

O MNSAN foi construído em XML por ser um padrão reconhecido internacionalmente para armazenar e trocar informações. Como a XML é auto descritiva, torna-se uma tarefa simples identificar e representar informações que sejam entendidas tanto pelo computador quanto por humanos (W3C, 2015). Com XML é possível expressar elementos por meio de marcações, conhecidas como tags, onde cada uma delas pode representar um determinado elemento do modelo.

Para validar e verificar a conformidade do MNSAN, foi utilizado a XSD (XML Schema Definition). Através da XSD é possível descrever a forma do conteúdo do documento, quais restrições devem ser adicionadas aos dados e também validar qual tipo de dado deverá ser aceito pelo modelo. O documento XML, validado pelo XSD, é aplicado como meio de representação dos dados referentes ao negócio assegurando que a informação recebida é completa e padronizada.

Para a realização do teste de viabilidade dos modelos, foram definidos critérios para cada parâmetro de comparação. O objetivo foi prover aplicações práticas e que se aproximem 
das necessidades e peculiaridades que uma situação de mercado pudesse apresentar. Cada um dos testes correspondia aos parâmetros estabelecidos para comparação, onde foram contrapostas as supostas vantagens do uso de um modelo semântico, contra a tentativa de reprodução do mesmo resultado, utilizando modelos não semânticos (QUADRO 1).

QUADRO 1- Capacidades dos modelos a serem testadas. Fonte: Do Autor

\begin{tabular}{|l|l|l|}
\hline \multicolumn{1}{|c|}{ Capacidade } & \multicolumn{1}{|c|}{ Objetivo } & \multicolumn{1}{c|}{ Pergunta de competência } \\
\hline Classificação & $\begin{array}{l}\text { Verificar se um dado conceito pode } \\
\text { ser sempre considerado um } \\
\text { subconjunto de outro conceito geral }\end{array}$ & $\begin{array}{l}\text { "Vestidos são sempre categorizados como produtos de moda } \\
\text { feminina?". }\end{array}$ \\
\hline $\begin{array}{l}\text { Verificação de } \\
\text { Instâncias }\end{array}$ & $\begin{array}{l}\text { Inferir se uma dada instância } \\
\text { pertence a determinado conceito }\end{array}$ & $\begin{array}{l}\text { "Dado o produto X, ele pode ser classificado como uma calça } \\
\text { com algum tamanho plus size?" }\end{array}$ \\
\hline $\begin{array}{l}\text { Verificação de } \\
\text { Consistência }\end{array}$ & $\begin{array}{l}\text { Verificar a consistência das } \\
\text { instâncias inseridas no modelo }\end{array}$ & $\begin{array}{l}\text { "Dado um determinado produto com preenchimento X. Qual é } \\
\text { o domínio pertencente a X?". }\end{array}$ \\
\hline $\begin{array}{l}\text { Verificação de } \\
\text { Realização }\end{array}$ & $\begin{array}{l}\text { Verificar a capacidade do modelo em } \\
\text { determinar quais são todos os } \\
\text { conceitos segundo os quais um } \\
\text { indivíduo pode ser classificado }\end{array}$ & $\begin{array}{l}\text { "Um determinado produto X que é categorizado tanto como de } \\
\text { categorizado como produto unissex?" }\end{array}$ \\
\hline $\begin{array}{l}\text { Verificação de } \\
\text { Recuperação }\end{array}$ & $\begin{array}{l}\text { Determinar todos os indivíduos que } \\
\text { pertencem a um dado conceito }\end{array}$ & $\begin{array}{l}\text { "Quais são todos os indivíduos que podem ser classificados } \\
\text { como calças que contenham algum tamanho plus size? “ }\end{array}$ \\
\hline $\begin{array}{l}\text { Redução da } \\
\text { Ambiguidade }\end{array}$ & $\begin{array}{l}\text { Investiga-se a capacidade de o } \\
\text { modelo ser robusto com relação a } \\
\text { ambiguidades }\end{array}$ & $\begin{array}{l}\text { "Dado um contexto onde os tamanhos de roupa possam ser } \\
\text { inseridos tanto no padrão norte americano quanto no padrão } \\
\text { brasileiro, ao buscar roupas que possuam determinado tamanho } \\
\text { norte americano, as equivalentes no padrão brasileiro também } \\
\text { serão informadas?" }\end{array}$ \\
\hline
\end{tabular}

\section{RESULTADOS E DISCUSSÃO}

A criação de um padrão genérico identificou algumas propriedades duplicadas. Para apresentar apenas uma única propriedade houve a mesclagem delas. Esta relação é apresentada noQUADRO 2 demonstrando a unificação das propriedades. Por exemplo, para a propriedade de "cor do produto" que aparece em ambos os documentos foi transformada em uma propriedade denominada "Preenchimento". Essa mudança ocorreu, pois existem produtos com informações tanto de cor e a de textura que está relacionado ao tipo de estampa.

QUADRO 2 - Relação das propriedades duplicadas com as propriedades resultantes. Fonte: Do Autor

\begin{tabular}{|l|l|} 
Documento de Origem & Propriedade Resultante \\
\hline
\end{tabular}




\begin{tabular}{|l|l|l|}
\hline \multicolumn{1}{|c|}{ produto.xml } & Produto.php & \\
\hline ID_PRODUTO & SKU & SKU \\
\hline NOME_PRODUTO & Nome & Nome \\
\hline DESCRICAO & Descricao & Descrição \\
\hline COR & Cor & Preenchimento \\
\hline MARCA & Marca & Marca \\
\hline PRECO & Preco & Preço \\
\hline PRECO_PROMO & PrecoPor & Preço Promoção \\
\hline URL & URL & URL \\
\hline URL_IMAGEM & Imagem & URL Imagem \\
\hline SUB_CLASSIFICACAO & SubClassificacao & Classificação \\
\hline CATEGORIA_PRINCIPAL & Categoria & Categoria \\
\hline SUB_CATEGORIA & SubCategoria & Subcategoria \\
\hline
\end{tabular}

Visando manter um grau de simplicidade no modelo padrão genérico as propriedades redundantes foram eliminadas. Por exemplo, as propriedades "COD_BARRA" e “ID_PRODUTO" possuem a mesma informação que representam unicamente um produto. E outro exemplo é a concatenação de propriedades sendo a "N_PARCELA" e "VALOR_PARCELA" que foram encontradas somente no documento XML, sendo consideradas importantes foram adicionadas como propriedades opcionais. Uma nova propriedade "tamanho" foi adicionada ao modelo genérico, pois foi identificado que para produtos de moda possui extrema importância no contexto de produtos de moda.

A relação hierárquica das propriedades é apresentada na FIGURA 1. A propriedade "Produto de Moda" e um Artefato de Negócio que contém todas as propriedades referentes a um produto de moda. "Produto de Moda" é uma propriedade filha de um conceito global denominado "Produto" que representa todas as espécies de produto que eventualmente poderiam ser modeladas. 


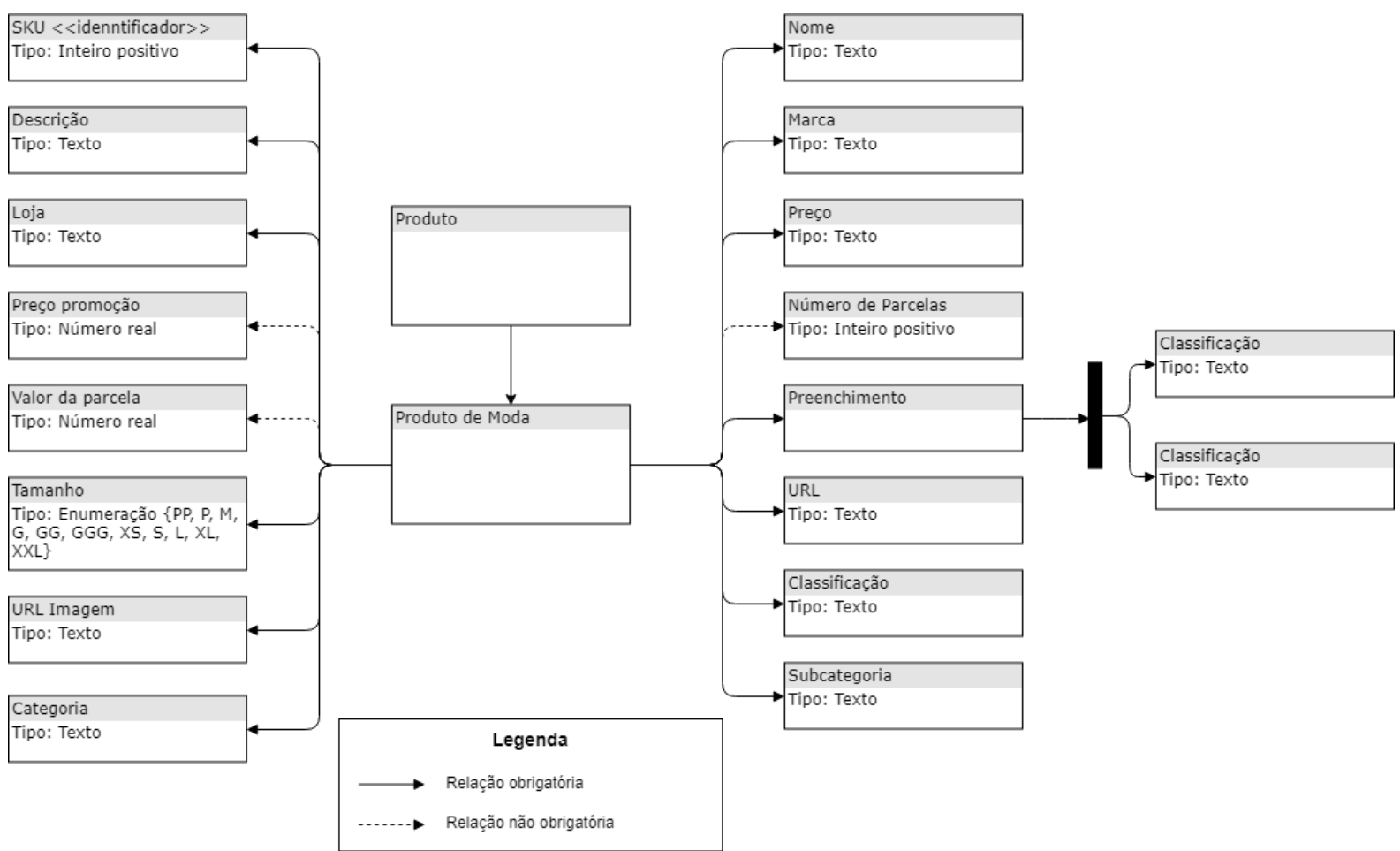

FIGURA 1 - Propriedades do padrão conceitual genérico. Fonte: Do Autor

Foi construído um modelo genérico do MNSAN para mapear os conceitos de representação em tags XML. A árvore de atributos é apresentada na FIGURA 2, onde o elemento "produto" é o elemento raiz. O elemento "produto-de-moda" é classificado como filho, representando as instâncias de cada produto de moda. Portanto, os elementos que estiverem entre suas tags de início e de fim fazem parte de um produto de moda.

Durante o processo de implantação dos elementos do MNSAN foram criadas restrições que tem como objetivo definir os tipos de dados para cada um dos elementos. Por exemplo, o "tamanho" é o identificador do produto de modas, com a restrição sobre a numeração das roupas. As numerações podem ser classificadas tanto nos padrões brasileiros quanto ao norte americano. 


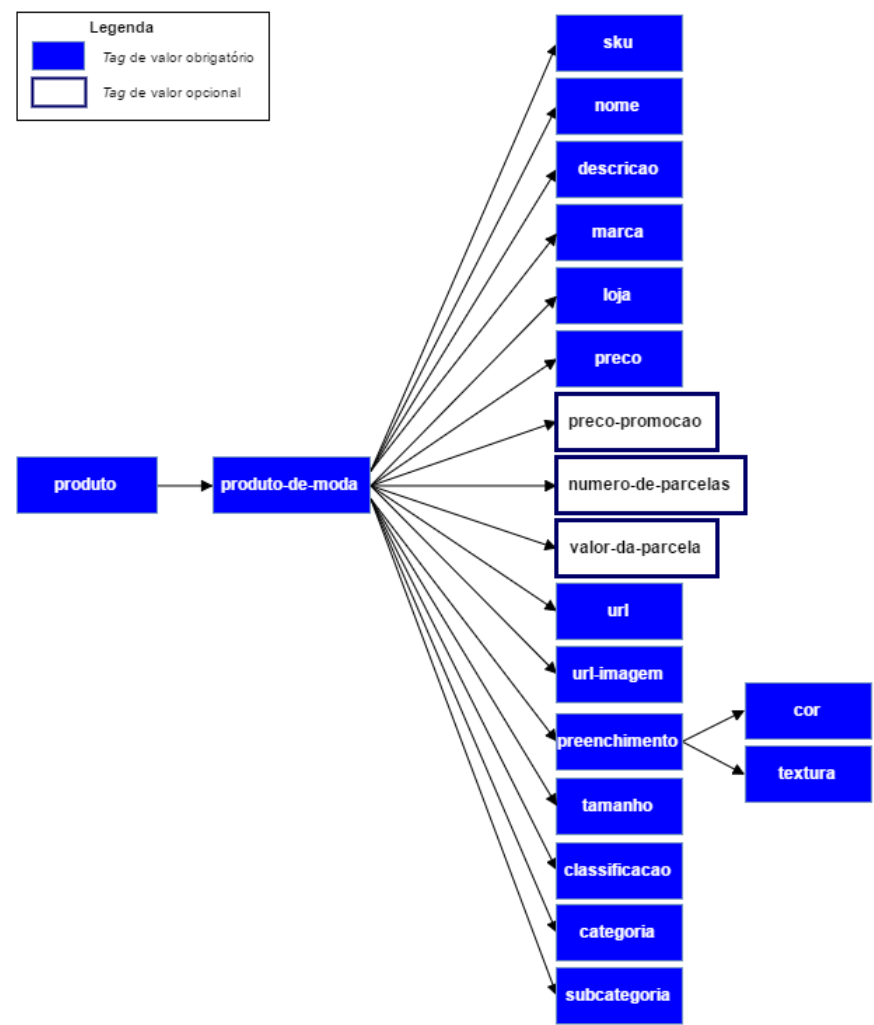

FIGURA 2 - Propriedades do padrão conceitual genérico. Fonte: Do Autor

A construção do MSAN com suas classes é apresentada na FIGURA 3. Todas as classes e subclasses foram consideradas dentro do conceito Thing. Desta forma é possível dizer que nenhum indivíduo pertença a duas classes distintas ao mesmo tempo e nenhuma relação de equivalência de indivíduos pode ocorrer entre elas.

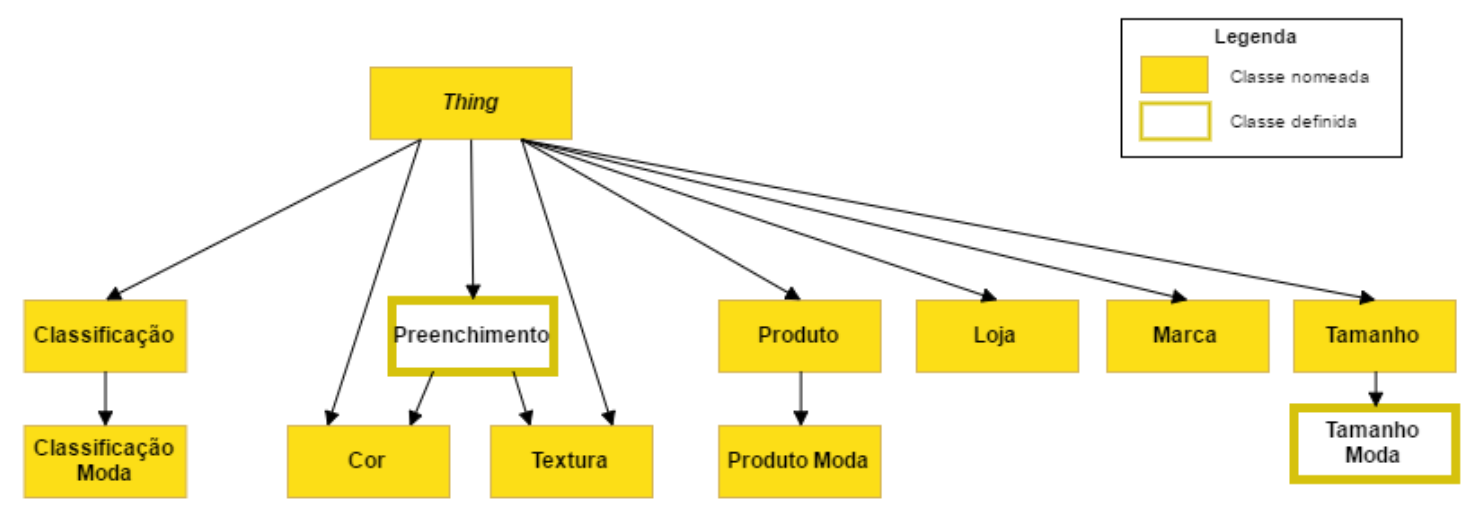

FIGURA 3 - Hierarquia de classes do MSAN genérico. Fonte: Do Autor

Para as relações entre propriedades de dados, foi necessário especificar a obrigatoriedade ou não das suas relações e o tipo de dado a que cada uma delas se relaciona. Já nas relações entre propriedades de objeto, além de especificar a obrigatoriedade das relações e 
as classes às quais cada uma delas se relaciona, também foi necessário adicionar axiomas de fechamento sobre elas, para restringir seu conjunto imagem.

Um exemplo de relações que foram aplicadas ao MSAN e que não puderam ser expressas no MSAN é com relação à classe definida “Tamanho Moda". Sua restrição estabelece quais são os indivíduos que pertencem a ela. Como feito para o MNSAN também se determinou alguns tamanhos referentes a produtos de moda, tanto no padrão norte americano quanto no padrão brasileiro. Para estabelecer as correspondências de tamanho entre os padrões, foi estabelecida sobre cada indivíduo a relação de equivalência com o seu correspondente.

A propriedade de dados "SKU" foi criada para ser um identificador único, não sendo possível ter ao mesmo tempo dois valores. Sendo uma propriedade funcional serve para indicar que determinados indivíduos são distintos, se eles possuem identificadores diferentes.

Para provar a viabilidade de uso do MNSAN e MSAN os modelos foram submetidos a testes de prova. Para todas as capacidades testadas, o MSAN se mostrou apto a inferir as informações sem necessitar alterações no modelo. Já o MNSAN requereu que o modelo fosse alterado para que as inferências fossem corretamente verificadas. O QUADRO 3 apresenta como os modelos se saíram nos testes, onde $\mathrm{X}$ representa que o modelo atendeu a pergunta de competência com sucesso.

QUADRO 3 - Hierarquia de classes do MSAN genérico. Fonte: Do Autor

\begin{tabular}{|l|c|c|}
\hline \multicolumn{1}{|c|}{ Capacidade } & MNSAN & MSAN \\
\hline Classificação & & $\mathrm{X}$ \\
\hline Verificação de Instância & & $\mathrm{X}$ \\
\hline Verificação de Consistência & & $\mathrm{X}$ \\
\hline Verificação de Realização & & $\mathrm{X}$ \\
\hline Verificação de Recuperação & & $\mathrm{X}$ \\
\hline Redução da Ambiguidade & & $\mathrm{X}$ \\
\hline
\end{tabular}

\section{CONCLUSÃO}

Com o mercado moderno buscando cada vez mais integração entre as empresas. Um processo de negócios bem definido proporciona um papel fundamental para que a empresa consiga se destacar e gerando valor agregado para sua organização. 
Portanto objetivou-se demonstrar a viabilidade técnica e as supostas vantagens da utilização de Artefatos de Negócio que possuam expressividade semântica, denominados MSAN. Esta utilização foi contraposta com a modelagem tradicional de artefatos, denominados MNSAN. O contraste das duas abordagens foi explicitado através de testes que deveriam provar as supostas vantagens da aplicação dos modelos semânticos. Vale ressaltar o caráter inovador deste estudo dada a evidente carência de estudos que demonstrem de forma prática as diferenças entre modelos ontológicos e não-semânticos, ainda mais aplicado a um cenário real de mercado.

Ao final conclui-se que existem vantagens notáveis provenientes da aplicação de modelos com expressividade semântica sobre aqueles meramente sintáticos, trazendo ganhos que contribuem para que estes sejam valiosos na modelagem de padrões de Artefatos de Negócio que visam a interoperabilidade entre empresas parceiras. Muito disto se deve à aptidão dos modelos ontológicos para expressar conceitos mais complexos com a garantia da consistência lógica. Esta consistência, aliada à qualidade das restrições e definições aplicadas sobre conceitos e indivíduos, por sua vez, é o que permite que inferências sobre o modelo possam ser realizadas e o conhecimento implícito manifestar-se explícito.

\section{REFERÊNCIAS}

ALMEIDA, Maurício Barcellos. Um modelo baseado em ontologias para representação da memória organizacional. 2006. Tese de Doutorado - Universidade Federal de Minas Gerais, Brasil, 2006.

CAFEZEIRO, Isabel et al. Ontologias: interoperabilidade, contexto, computação ubíqua. Seminário de Pesquisa em Ontologia no Brasil, Niterói. Disponível em:< http://www.lbd.dcc.ufmg.br/colecoes/ontobras/2008/006. PDF, 2008 .

CIOCOIU, Mihai; NAU, Dana S; GRUNINGER, Michael. Ontologies for integrating engineering applications. Journal of Computing and Information Science in Engineering, v. 1, n. 1, p. 12-22, 2001.

FONSECA, Frederico; EGENHOFER, Max; BORGES, Karla AV. Ontologias e interoperabilidade semântica entre SIGs. 2000, [S.1: s.n.], 2000.

GASSEN, Jonas Bulegon. Modelagem de processos de negócio: rótulos e ontologias. 2014. 112 f. Universidade Federal do Rio Grande do Sul, Porto Alegre, 2014. Disponível em: <https://lume.ufrgs.br/handle/10183/107104>.

HERAVI, Bahareh Rahmanzadeh. Ontology-based information standards development. 2012. PhD Thesis Brunel University, School of Information Systems, Computing and Mathematics, 2012.

KAMADA, Aqueo. Interoperabilidade semântica. 2011.

LIMA, Júnio César; CARVALHO, Cedric L. Uma Visão da Web Semântica. [S.1.]: Technical Report INF_001/94, Instituto de Informática-Universidade Federal ..., 2004.

LIU, Rong; BHATTACHARYA, Kamal; WU, Frederick Y. Modeling business contexture and behavior using business artifacts. 2007, [S.1.]: Springer, 2007. p. 324-339.

NETO, Manoel Veras Sousa; JUNIOR, Josué Vitor Medeiros. Afinal, o que é Business Process Management (BPM)? Um novo conceito para um novo contexto. Revista eletrônica de sistemas de informação, v. 7, n. $2,2008$. 
OLIVEIRA, Rafael Bruno Cavalhero de. Uma metodologia de modelagem de processos de negócio orientada à gestão da informação e do conhecimento. Perspectivas em Ciência da Informação, v. 15, n. 1, p. 319-320, 2010.

ROSPOCHER, Marco et al. Collaborative Enterprise Integrated Modelling. In: PROCEEDINGS OF THE 5TH INTERNATIONAL WORKSHOP ON SEMANTIC WEB APPLICATIONS AND PERSPECTIVES, 2008, Rome, Italy. Anais... Rome, Italy: [s.n.], 2008.

SOUZA, Renato Rocha; ALVARENGA, Lídia. A Web Semântica e suas contribuições para a ciência da informação. Ciência da Informação, v. 33, n. 1, 2004.

W3C. OWL - Semantic Web Standards. Disponível em: 〈https://www.w3.org/2001/sw/wiki/OWL〉. Acesso em: 6 jun. 2015.

W3C. World Wide Web Consortium (W3C). Disponível em: <https://www.w3.org/>. Acesso em: 6 jun. 2015. 